\title{
Effects of Extremely Low Frequency Magnetic Field on the Secondary Structures of $\beta$-Amyloid and Human Serum Albumin
}

\author{
Saqer Mohamad Darwish ${ }^{1,}$ *, Husain Rashad Alsamamra ${ }^{1}$, Sawsan Eid Abusharkh ${ }^{1}$, \\ Imtiaz Mohammed Khalid ${ }^{2}$, Rania Abdeljalil Alfaqeh ${ }^{1}$, Musa Mahmoud Abuteir ${ }^{1}$ \\ ${ }^{1}$ Physics Department, Al-Quds University, Abu-Dies, Jerusalem, Palestine \\ ${ }^{2}$ Chemistry Department, Birzeit University, Birzeit, Palestine
}

Email address:

sdarwish@staff.alquds.edu (S. M. Darwish), hsamamra@staff.alquds.edu (H. R. Alsamamra), sabusharkh@staff.alquds.edu (S. E. Abusharkh), ikhalid@birzeit.edu (I. M. Khalid), raniafaqeh@gmail.com (R. A. Alfaqeh), abuteir@staff.alquds.edu (M. M. Abuteir)

${ }^{*}$ Corresponding author

\section{To cite this article:}

Saqer Mohamad Darwish, Husain Rashad Alsamamra, Sawsan Eid Abusharkh, Imtiaz Mohammed Khalid, Rania Abdeljalil Alfaqeh, Musa Mahmoud Abuteir. Effects of Extremely Low Frequency Magnetic Field on the Secondary Structures of $\beta$-Amyloid and Human Serum Albumin. European Journal of Biophysics. Vol. 5, No. 6, 2017, pp. 89-103. doi: 10.11648/j.ejb.20170506.11

Received: November 28, 2017; Accepted: December 12, 2017; Published: January 10, 2018

\begin{abstract}
Human serum albumin and $\beta$-amyloid were exposed to extremely low frequency (ELF) magnetic field of $1.5 \mathrm{mT}$ intensity and $50 \mathrm{~Hz}$ frequency. The effects of exposure were investigated in the mid-infrared region by means of Fourier selfdeconvolution spectroscopic analysis. The experimental results suggest that exposure to the ELF magnetic field has reversible effects on the out of phase combination of $\mathrm{N}-\mathrm{H}$ in plane bending and $\mathrm{C}-\mathrm{N}$ stretching vibrations of the secondary structures of the two proteins. The exposure of $\beta$-amyloid and human serum albumin to ELF magnetic field affected the absorption spectra of the vibration bands by changes in peak positions for the amide II bands and changes of intensities in most of the bands in the amide I and amide II regions. In the fingerprint region, the most sensitive vibrations to the magnetic field are found to be in the (720-600) $\mathrm{cm}^{-1}$ range. After removing the magnetic field, it took the vibration bands more than 10 minutes of a gradual change toward returning to their original spectra, obtained before the exposure. It is suggested that hydrogen bonds can alter the frequency of a stretching vibration depending on the increase or decrease of strain on the vibrations.
\end{abstract}

Keywords: FTIR-Spectroscopy, ELF-Magnetic Field, $\beta$-Amyloid, HAS, Protein Dynamics

\section{Introduction}

Infrared spectroscopy is one of the most important analytical techniques recognized in obtaining information on structural features of proteins. The excitation of molecular vibrations occurs when the frequency of the absorbed infrared radiation is equal to the difference between the corresponding vibrational energy levels. Fourier transform infrared spectroscopy (FTIR) spectroscopy is a valuable tool in measuring wavelength and intensity of the absorption of a protein sample by IR.

A considerable amount of scientific research had shown a strong correlation between band positions of the IR spectra and the secondary structure of the studied proteins. The frequencies of the observed absorption bands in several proteins are linked to certain molecular vibrations in the secondary structures, such as $\alpha$-helical or $\beta$-sheet structures [1-3]. Normal functionality of a protein depends on the three-dimensional structures of its constituents of peptides and polypeptide.

There are nine IR bands, named amide A, amide B and amides I-VII, in the order of decreasing frequency [3, 4]. The most intense and most useful for the analysis of the secondary structure of proteins was found to be the amide I band $\left(1600-1700 \mathrm{~cm}^{-1}\right)$, which involves $\mathrm{C}=\mathrm{O}$ stretching vibration $[4,5]$. The amide II band (1480-1600) $\mathrm{cm}^{-1}$ and amide III band $(1220-1320) \mathrm{cm}^{-1}$ contribute greatly to studies despite of the complex nature of their compositions 
and can be used for secondary structure prediction [6-8]. The spectra in the region from about $(1300-500) \mathrm{cm}^{-1}$ arise due to complicated combinations of $(\mathrm{C}-\mathrm{C}, \mathrm{C}-\mathrm{O}, \mathrm{C}-\mathrm{N})$ stretching and bending vibration. This range, referred to as the fingerprint region, is important because each compound produces its unique pattern of peaks.

Within the cells, proteins must fold into the specific conformational state in a complex highly crowded environment aided by a range of auxiliary proteins [9, 10]. Changes in protein properties and enzyme reactions have been studied by analyzing secondary structures of proteins by IR spectroscopy. The regions of IR spectra corresponding to the amide I band, the amide II band, and amino acids side chain vibrations are especially suitable targets of such analysis.

$\beta$-amyloid $(\mathrm{A} \beta)$ which contains rich structured $\beta$-sheet, is insoluble in the physiological solutions and forms toxic oligomers within the human body in general leading to amyloidosis in different diseases [11-13]. Human serum albumin (HSA) is highly abundant protein in the blood plasma and usually binds and transports fatty acids, hormones, bilirubin, tryptophan, steroids, metal ions, and therapeutic agents. HSA serves as the major soluble protein constituent of the circulatory system, it contributes to colloid osmotic blood pressure, and it can bind and carry drugs which are poorly soluble in water [14].

Several studies have indicated a relationship between the exposure to extremely low frequency (ELF) electromagnetic fields and an increased risk of cancer $[15,16]$. The world Health organization (WHO), had reported in 2007 that external ELF magnetic fields induce currents in the body and can lead to nerve and muscle stimulation in nerve cells. In 2002 The International Agency of Research on Cancer (IARC) had classified ELF magnetic fields as "possibly carcinogenic to humans" [17-21]. Although magnetic particles are found in the human body in various forms, not enough evidence appear to confer a sensitivity to environmental ELF magnetic fields. It seems that further scientific investigations are needed to explore any possible indirect effect under certain conditions.

The interest in the effect of ELF magnetic fields on living cells is significant for two reasons, first is the potential of its harmful implications on human health and the second is due to its vital role of sustaining biological functions for animals and humans. The effects on some biological processes, such as photosynthetic reaction of cryptochrome protein have demonstrated change in the protein orientations with respect to earth magnetic field causing it to act as a magnetic compass. In general, each of cryptochrome and photolyase can respond to magnetic field and transduce a physical signal into a chemical signal [22-28]. This magnetoreception in which an organism detects a magnetic field for navigating a location, is based on Zeeman's quantum effect where unpaired electron spins may be either antiparallel $(\uparrow \downarrow)$, corresponding to a singlet state, $(\mathrm{S})$ or parallel $(\uparrow \uparrow)$ which correspond to a triplet state, $(\mathrm{T})$ are created as a result of light absorption in the presence of applied magnetic field [29-35].

Magnetic field may affect some proteins through the electrostatic interactions between electrons. It is possible to account for the anomalous Zeeman effect where weak magnetic field can yield a magnetic dipolar energy of $(E=g$ $\mathrm{m}_{\mathrm{j}} \mu_{\mathrm{B}} \mathrm{B}$ ), where $\mathrm{g}$ is the Lande factor, $\mathrm{B}$ is the magnetic field, $\mathrm{m}_{\mathrm{j}}$ is the quantum number for the total angular momentum and $\mu_{\mathrm{B}}$ is Bohr magnetron. If $\left(\mu_{\mathrm{B}} . \mathrm{B}\right)$ is less than the spin orbit energy this will split the energy for the $p$ and $s$ states and spectral lines due to electric dipole transition will become allowed. If the Hamiltonian for two electron system commutes with the particle exchange operator then the degeneracy will be lifted creating $\mathrm{S}=0$ state (singlet state) and $\mathrm{S}=1$ (triplet state).

Charge motion and therefore magnetic fields are part of our environment at every level which leads to a natural assumption that magnetic field have a vital role in all biological systems and their functions. In this work, we intend to investigate the effects of exposing A $\beta$ and HSA to extremely low frequency electromagnetic field.

\section{Materials and Methods}

$A \beta$ (1-40) human, in powder form and human serum albumin HSA (fatty acid free) were purchased from Sigma Aldrich chemical company and used without any further purification.

\subsection{Preparation of Stock Solutions}

An amount of $0.5 \mathrm{mg}$ of $\mathrm{A} \beta$ (1-40) was dissolved in 250 $\mu \mathrm{L}$ of triple distilled water to give a concentration of 0.462 $\mathrm{mM}$ as a stock solution.

HSA was dissolved in phosphate buffered saline (40 $\mathrm{mg} / \mathrm{ml}$ ). The concentration of HSA in the buffer solution was prepared using its list molecular weight of $66.5 \mathrm{kDa}$. The solutions of $\mathrm{A} \beta$ and HSA were incubated for $1 \mathrm{~h}$ (at $20^{\circ} \mathrm{C}$ ) before spectroscopic measurements were taken.

\subsection{FTIR Spectroscopic Measurements}

The FTIR measurements were obtained on a Bruker IFS 66/S spectrophotometer equipped with a liquid nitrogencooled MCT detector and a $\mathrm{KBr}$ beam splitter. The spectrometer was continuously purged with dry air during the measurements. FTIR Samples were prepared after one hour of incubating the A $\beta$ and HSA free solutions at room temperature. Four drops of each sample were placed on a certain area on a silicon window plate and left to dry at room temperature.

A spectrum was taken as an average of 60 scans to increase the signal to noise ratio, and the spectral resolution was at $4 \mathrm{~cm}^{-1}$. The aperture used in this study was $8 \mathrm{~mm}$, which was found to give the best signal to noise ratio. Baseline correction, normalization and peak areas calculations were performed for all the spectra by OPUS software. The peak positions were determined using the second derivative by OPUS software in addition to the Fourier self-deconvolution (FSD) procedure which is kept at 6 steps for all deconvolution process to be consisted for data 
analysis. The concept of (FSD) assumes that a spectrum is composed of several single bands (each narrow band is characteristic of a secondary structure), which are broadened in the liquid or solid state.

The FTIR spectra of $A \beta$ and HSA were obtained in the featured region of $(4000-600) \mathrm{cm}^{-1}$. The FTIR spectra were acquired by subtracting the absorption spectrum of the buffer solution from the spectrum of the protein solution. For the net interaction effect, the difference spectra were generated using the featureless region of the $A \beta$ and HSA protein solutions $(1800-2200) \mathrm{cm}^{-1}$ as an internal standard [5]. The accuracy of this subtraction method was tested using several control samples with the same protein concentrations, which resulted into a flat base line formation. The spectral differences were used to investigate the net effect of ELF magnetic field on $A \beta$.

\subsection{ELF Magnetic Field Measurements}

A magnetic field with a sinusoidal waveform was generated by an electromagnet coil linked to an AC voltage regulating up to 220 volts, at the frequency of $50 \mathrm{~Hz}$. This device was used to generate time-varying ELF electromagnetic fields. This set up is used to expose the samples to a fixed magnetic field of $1.5 \mathrm{mT}$. The magnetic field was continuously monitored by a magnetic field axial probe PHYWE Gauss meter. In the last experimental part, each of $A \beta$ and HSA samples were exposed to ELF separately for 10 minutes and after removing the magnetic field, the spectra were taken every 2 minutes to monitor changes as a function of time.

\section{Experimental Results}

The FTIR absorption spectra are recorded in the range (4000-600) $\mathrm{cm}^{-1}$ including all the amide regions and the fingerprint region. The obtained spectra are taken for each of $\mathrm{A} \beta$ and HSA samples before and after exposure to ELF magnetic fields of $1.5 \mathrm{mT}$.

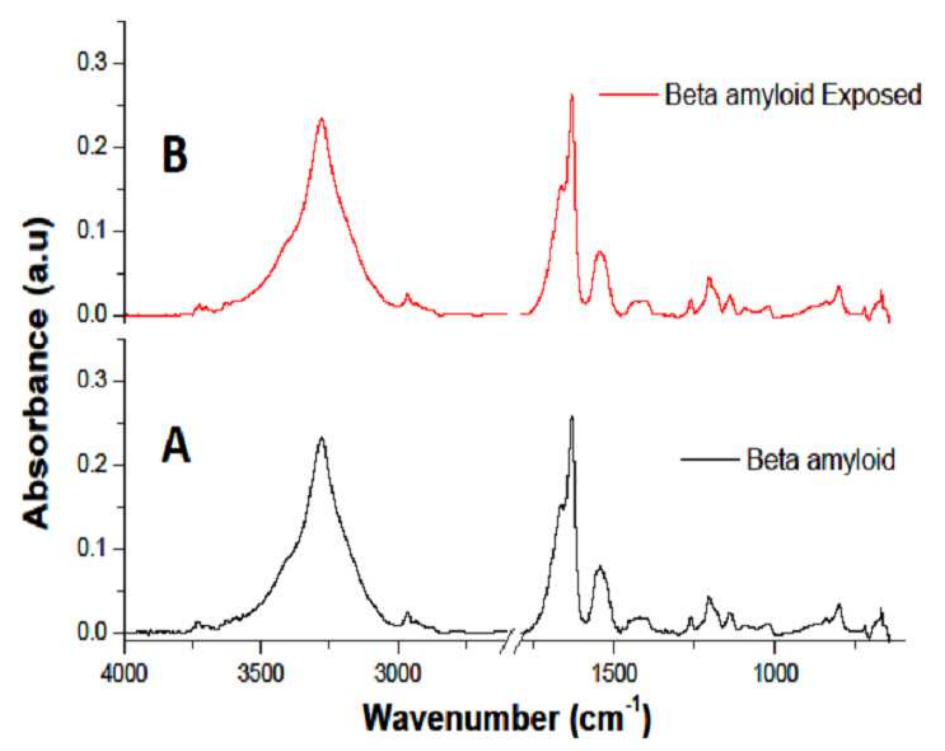

Figure 1. Absorption spectra of $\beta$-Amyloid (A) Sample is not exposed to ELF magnetic field, (B) Sample is exposed to ELF magnetic field 1.5 mT.

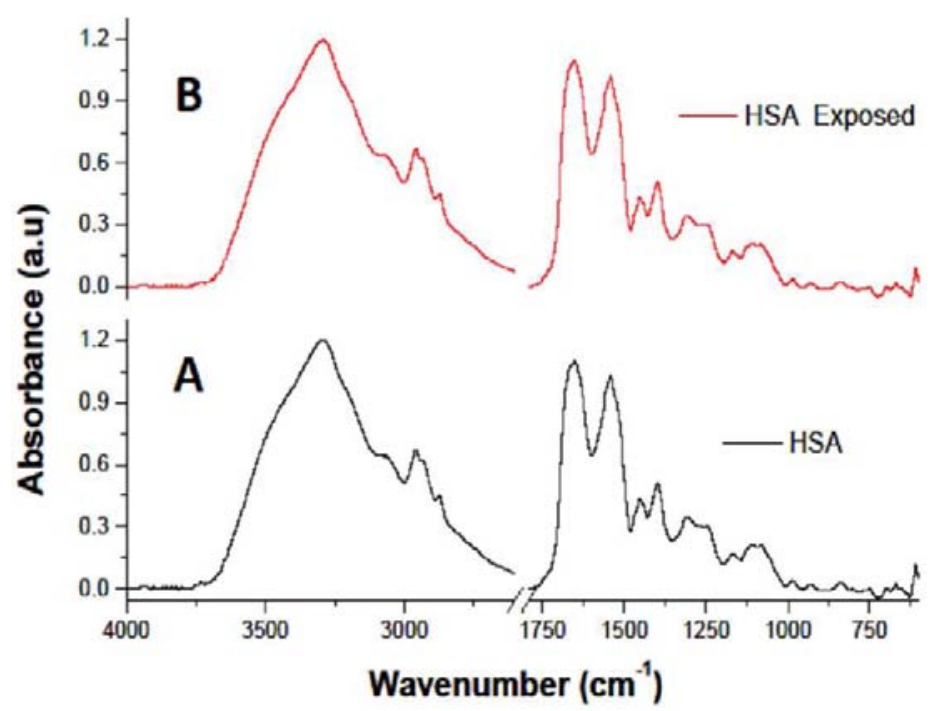

Figure 2. Absorption spectra of HSA (A) Sample is not exposed to ELF magnetic field, (B) Sample is exposed to ELF magnetic field $1.5 \mathrm{mT}$. 
The absorption spectra of $\mathrm{A} \beta$ and HSA are shown in figures 1 and 2, respectively. The spectra is shown for each of $A \beta$ and HSA before and after exposure to magnetic field. Each of the graphs shows the major absorption regions, including the amide bands and the fingerprint regions.

The second derivatives of the absorption spectra in the region of $(1800-600) \mathrm{cm}^{-1}$ are shown in figure 3 for both of HSA and A $\beta$. All major peaks have been identified based on the second derivative results and FSD spectra [36].

The analysis of the absorption spectra of $A \beta$ in the amide I region (1600 -1700) $\mathrm{cm}^{-1}$ by FSD, yields several bands. The peaks of these bands correspond to the $\mathrm{C}=\mathrm{O}$ stretching vibrations of the amide group, coupled to the $\mathrm{C}-\mathrm{N}$ stretching and $\mathrm{C}-\mathrm{C}-\mathrm{N}$ deformation mode [37]. On the other hand, the FSD spectra shows the individual bands in the amide II (1600 -1480) $\mathrm{cm}^{-1}$, which are mainly due to out of phase combination of $\mathrm{N}-\mathrm{H}$ in plane bending and $\mathrm{C}-\mathrm{N}$ stretching vibration modes [38]. As for amide III (1320-1220) $\mathrm{cm}^{-1}$, the absorption bands are mainly due to in-phase combination of the $\mathrm{N}-\mathrm{H}$ bending and the $\mathrm{C}-\mathrm{N}$ stretching vibration with some contributions from the $\mathrm{CO}$ in plane bending and the $\mathrm{CC}$ stretching vibration $[38,39]$.

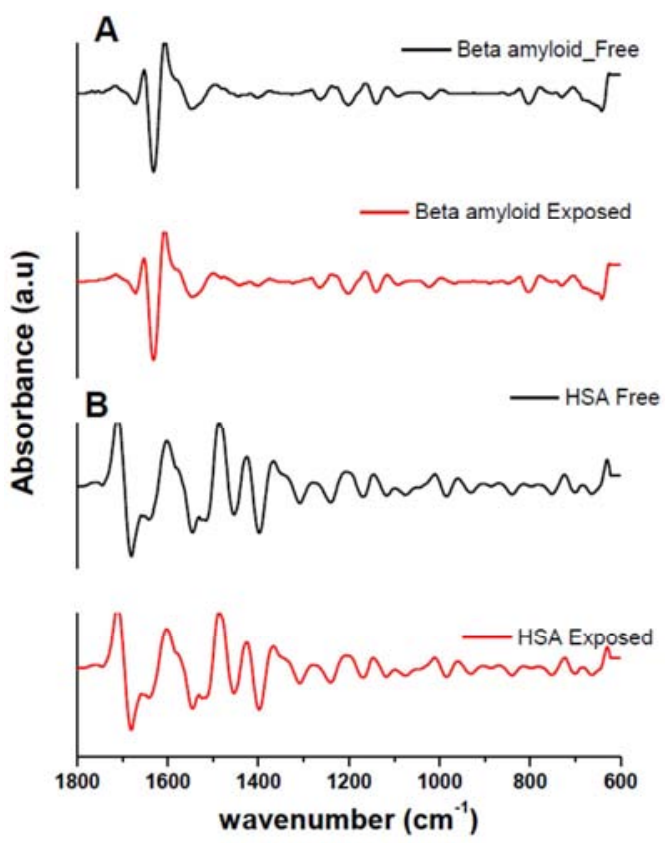

Figure 3. Second Derivative of Spectra of (A) Beta amyloid and (B) HSA.

Table 1. Bands assignment of the absorbance spectra $\left(\mathrm{cm}^{-1}\right)$ for $\beta$-amyloid in Amide (I, II, III) regions.

\begin{tabular}{|c|c|c|c|c|}
\hline \multirow{2}{*}{$\begin{array}{l}\text { Region } \text { cm }^{-1} \\
\text { Amide I (1600-1700) }\end{array}$} & \multicolumn{2}{|c|}{ Band positions } & \multirow{2}{*}{$\begin{array}{l}\text { Exposed to Field } \\
1609\end{array}$} & \multirow{2}{*}{$\begin{array}{l}\text { Difference spectra } \\
1612\end{array}$} \\
\hline & 1609 & $\beta$-sheet & & \\
\hline & 1629 & $\beta$-sheet & 1629 & 1628 \\
\hline & 1648 & Random & 1645 & 1642 \\
\hline & 1666 & $\alpha$-helix & 1664 & 1660 \\
\hline & 1682 & $\beta$-turn & 1680 & 1676 \\
\hline & 1697 & $\beta$-sheets & 1695 & 1692 \\
\hline \multirow[t]{7}{*}{ Amide II (1480-1600) } & 1490 & $\beta$-sheets & 1496 & 1499 \\
\hline & 1508 & Random & 1520 & 1515 \\
\hline & 1522 & $\beta$-sheets & 1532 & 1532 \\
\hline & 1540 & $\alpha$-helix & 1553 & 1550 \\
\hline & 1556 & $\alpha$-helix & 1569 & 1566 \\
\hline & 1573 & $\beta$-turn & 1585 & 1584 \\
\hline & 1594 & $\beta$-sheets & 1596 & 1599 \\
\hline \multirow[t]{5}{*}{ Amide III (1220-1330) } & 1242 & $\beta$-sheets & 1242 & 1242 \\
\hline & 1261 & Random & 1261 & 1263 \\
\hline & 1279 & $\beta$-turn & 1280 & 1279 \\
\hline & 1300 & $\alpha$-helix & 1300 & 1295 \\
\hline & 1318 & $\alpha$-helix & 1319 & 1308 \\
\hline
\end{tabular}

Table 2. Bands assignment of the absorbance spectra $\left(\mathrm{cm}^{-1}\right)$ for HSA in Amide (I, II, III) regions.

\begin{tabular}{|c|c|c|c|c|}
\hline \multirow{2}{*}{$\begin{array}{l}\text { Region } \text { cm }^{-1} \\
\text { Amide I (1600-1700) }\end{array}$} & \multicolumn{2}{|c|}{ Peak positions } & \multirow{2}{*}{$\begin{array}{l}\text { Exposed to Field } \\
1616\end{array}$} & \multirow{2}{*}{$\begin{array}{l}\text { Difference spectra } \\
1614\end{array}$} \\
\hline & --- & $\beta$-sheet & & \\
\hline & 1633 & $\beta$-sheet & 1633 & 1630 \\
\hline & 1651 & $\alpha$-helix & 1651 & 1645 \\
\hline & 1667 & $\beta$-turn & 1667 & 1660 \\
\hline & 1682 & $\beta$-turn & 1682 & 1676 \\
\hline & 1695 & $\beta$-sheets & 1695 & 1692 \\
\hline \multirow[t]{7}{*}{ Amide II (1480-1600) } & 1494 & $\beta$-sheets & 1494 & 1499 \\
\hline & 1511 & Random & 1511 & 1515 \\
\hline & 1521 & $\beta$-sheets & 1521 & 1532 \\
\hline & 1540 & $\alpha$-helix & 1540 & 1549 \\
\hline & 1556 & $\beta$-turn & 1556 & 1566 \\
\hline & 1572 & $\beta$-turn & 1572 & 1584 \\
\hline & 1591 & $\beta$-sheets & 1590 & 1600 \\
\hline \multirow[t]{2}{*}{ Amide III (1220-1330) } & 1225 & $\beta$-sheets & 1225 & 1232 \\
\hline & 1242 & $\beta$-sheets & 1242 & 1262 \\
\hline
\end{tabular}




\begin{tabular}{lllll}
\hline Region $\mathbf{~ c m}^{-1}$ & Peak positions & & Exposed to Field & Difference spectra \\
\hline 1269 & Random & 1267 & 1278 \\
& & & 1388 & 1293 \\
& 1388 & $\beta$-turn & 1298 & 1306 \\
-1298 & $\alpha$-turn & 1316 & -- \\
\hline
\end{tabular}

The amide I, amide II, and amide III bands are assigned in tables 1 and 2 based on several previous studies [40-44]. Furthermore, other bands in (1300-900) $\mathrm{cm}^{-1}$ range are assigned to $\mathrm{C}-\mathrm{O}$ bending modes of saccharides (glucose, lactose and glycerol), the peaks at (1360-1430) $\mathrm{cm}^{-1}$ attributed to vibrations of certain amino acids chains and (1430-1480) $\mathrm{cm}^{-1}$ is attributed to fatty acids, phospholipids and triglycerides $[45,46]$.

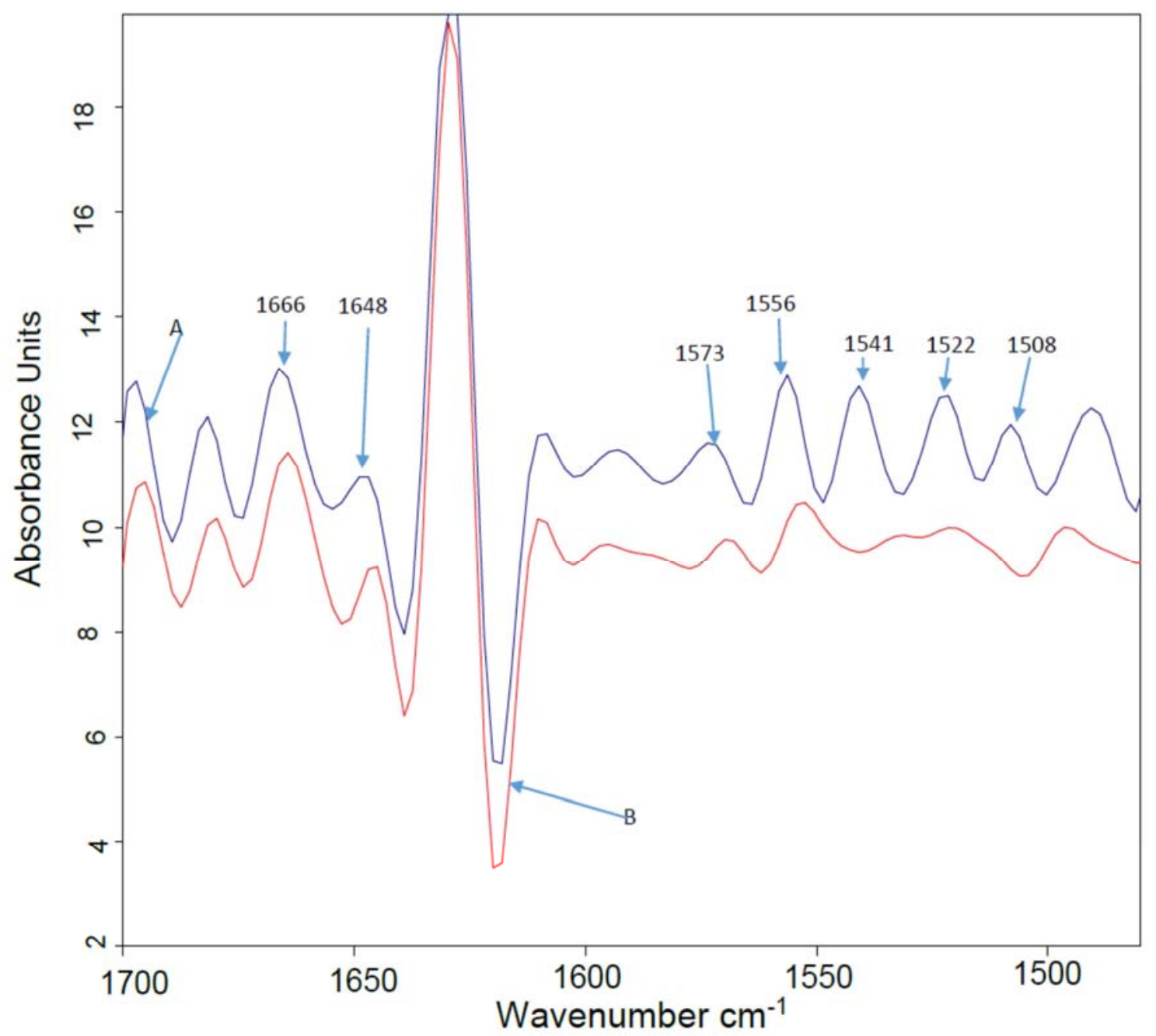

Figure 4. Deconvoluted absorption spectra for $\beta$-amyloid in the Amide I and Amide II regions.

A- $\beta$-amyloid free

B- $\beta$-amyloid exposed to ELF magnetic field $1.5 \mathrm{~m} \mathrm{~T}$ 


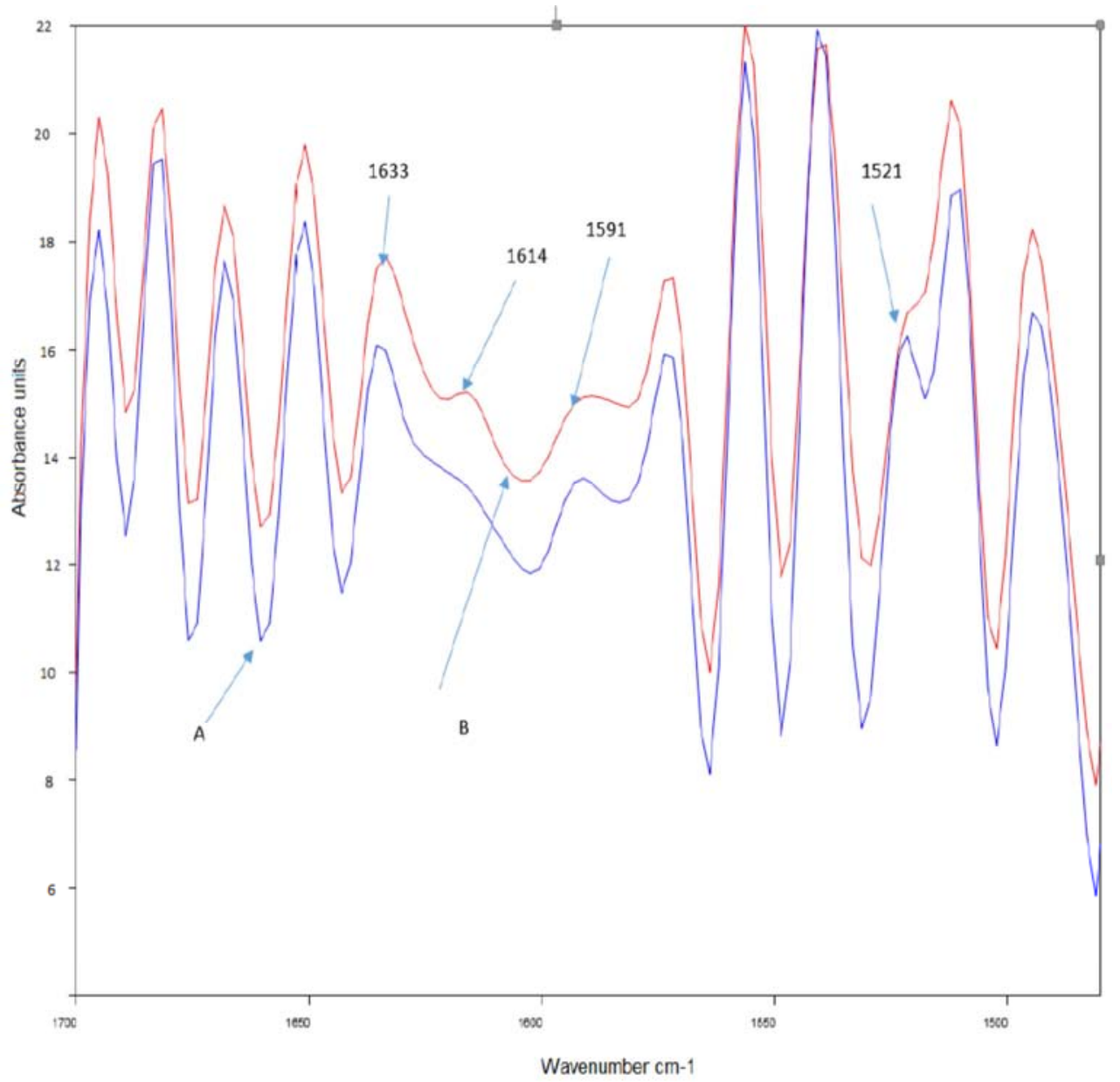

Figure 5. FSD spectra of HSA in the Amide I and Amide II regions.

A - Sample is not exposed to magnetic field

B- Sample is exposed to $1.5 \mathrm{mT}$ ELF magnetic field

The effect of exposing $\mathrm{A} \beta$ and HSA to a $50 \mathrm{~Hz}$ oscillating magnetic field of $1.5 \mathrm{mT}$ are shown in figures 4 and 5 for the amide I and amide II regions. The two figures show the FSD spectra for the two samples with all the main peaks that may reflect changes on the secondary structure of the two proteins. In the case of $\mathrm{A} \beta$, figure 4 shows the peaks' changes as listed in table 1 , where peak positions and band intensities have been affected. On the other hand, figure 5 shows the changes for the HSA sample after exposure to the same magnetic field, and all peaks are listed in table 2 .

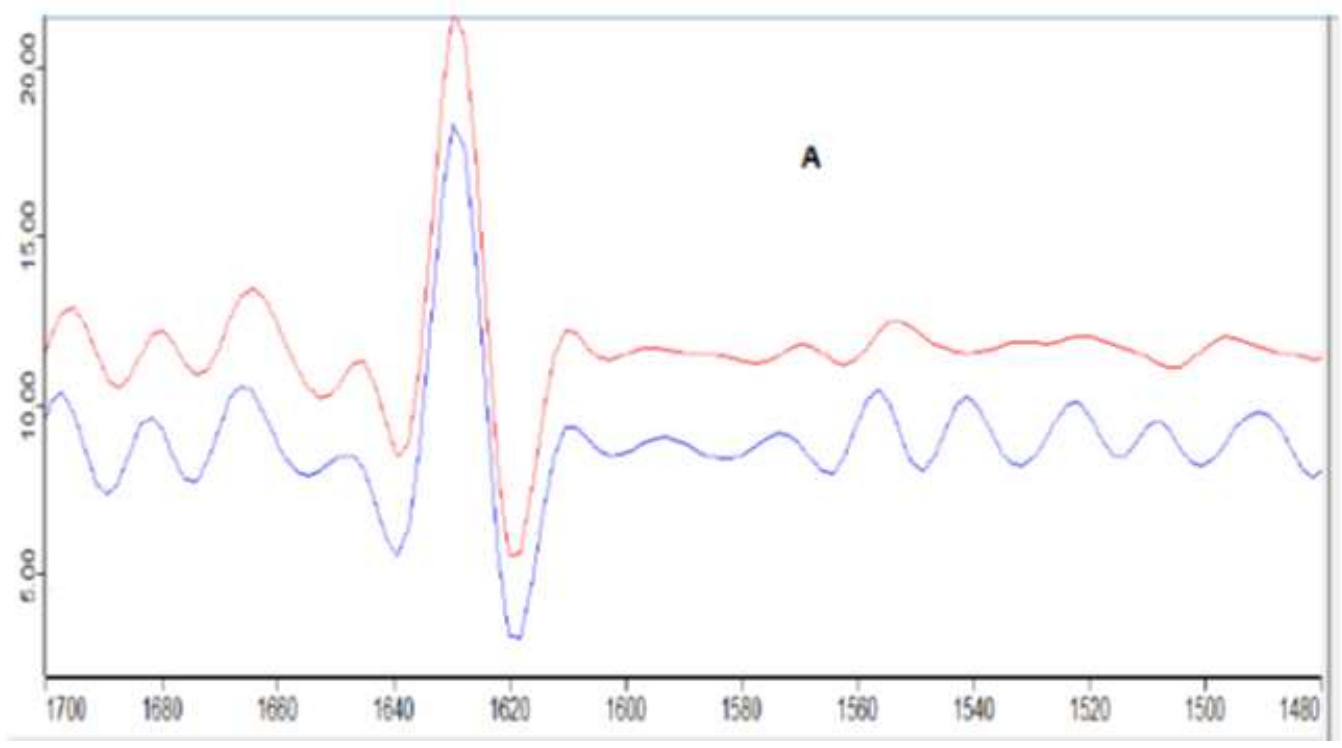




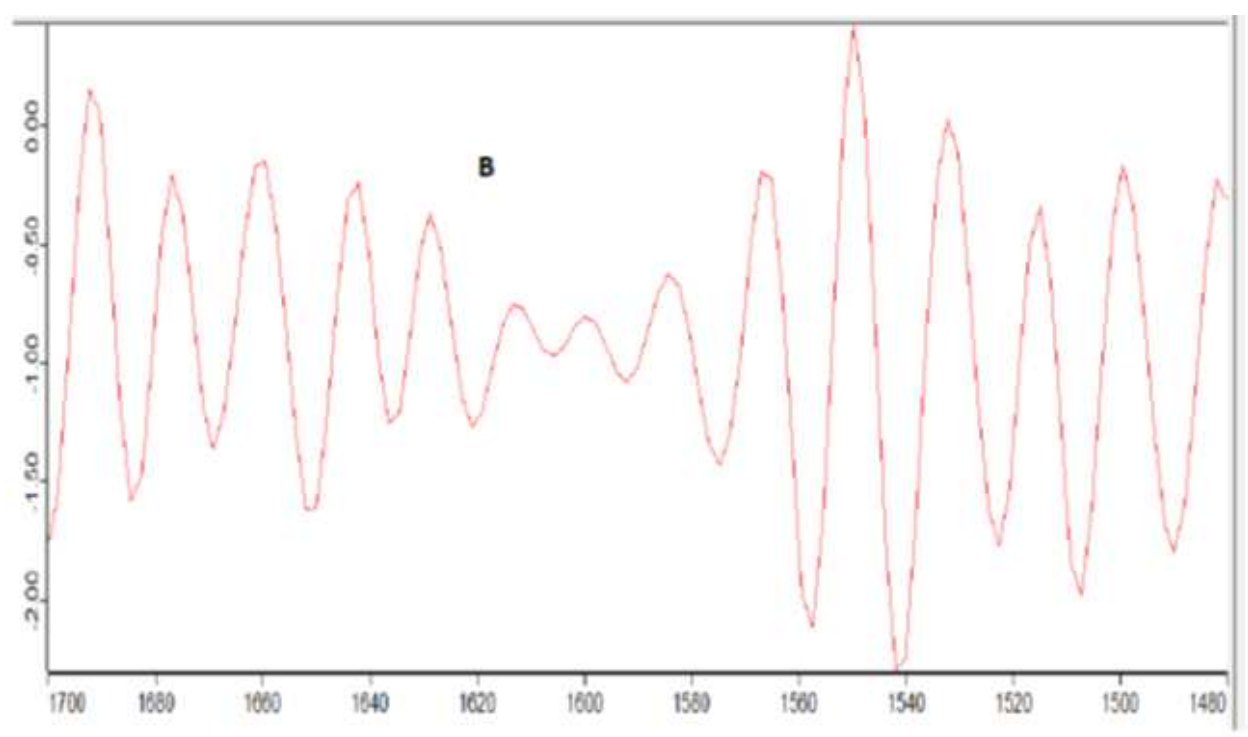

Wavenumber $\mathrm{cm}^{-1}$

Figure 6. Difference of FSD spectra of $\beta$-Amyloid.

A- The two FSD spectra exposed to magnetic field (above) and no field (below)

B- The difference of FSD spectra ( $\beta$-Amyloid exposed to magnetic field - $\beta$-amyloid without magnetic field)

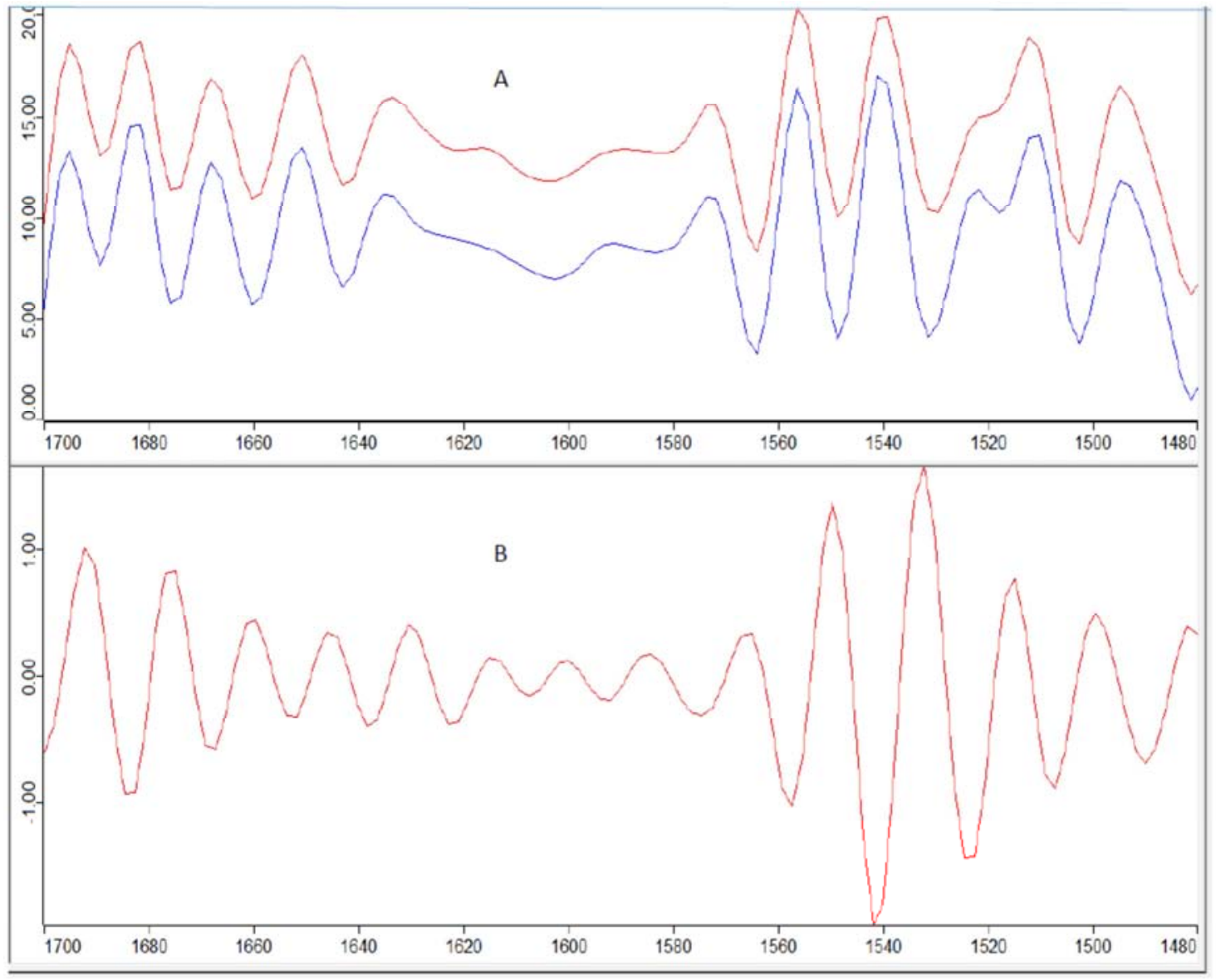

Wavenumbers $\mathrm{cm}^{-1}$

Figure 7. Difference of FSD spectra for HSA.

A- The two FSD spectra exposed to magnetic field (above) and no field (below)

B- The difference of FSD spectra (HSA exposed to magnetic field - HSA without magnetic field) 
The differences in the FSD spectra for each of $\mathrm{A} \beta$ and HAS before and after exposure are shown in figures 6 and 7 , respectively. Figure 6 shows the difference spectra which results from: $\{\mathrm{FSD}$ spectra of $\mathrm{A} \beta$ exposed to magnetic field - the FSD spectra of $A \beta\}$. The difference represents the net effect of the magnetic field on $A \beta$ as shown in figure 6-B, where it indicates that the majority of the observed peaks have been affected in different ways: some show increase of intensity, others shifted in their peak positions. These variations in change pertaining to $A \beta$ have been listed in table 1 . Similarly, changes for the HSA sample in the same range are shown in figure 6 and variations in changes are listed in table 2 .

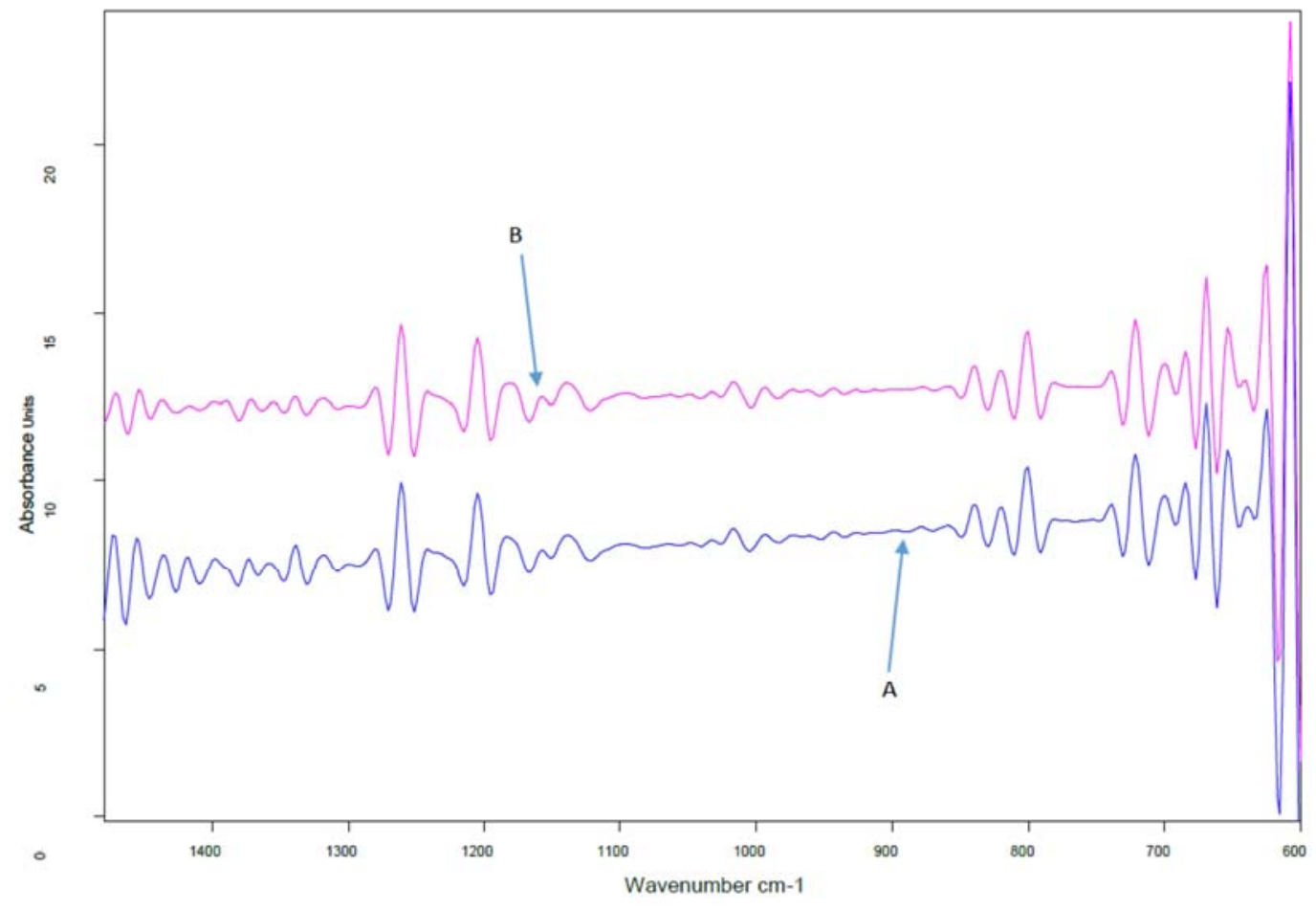

Figure 8. FSD spectra of AB before and after exposure to ELF magnetic field.

A- Before exposure to magnetic field

B- After exposure to magnetic field

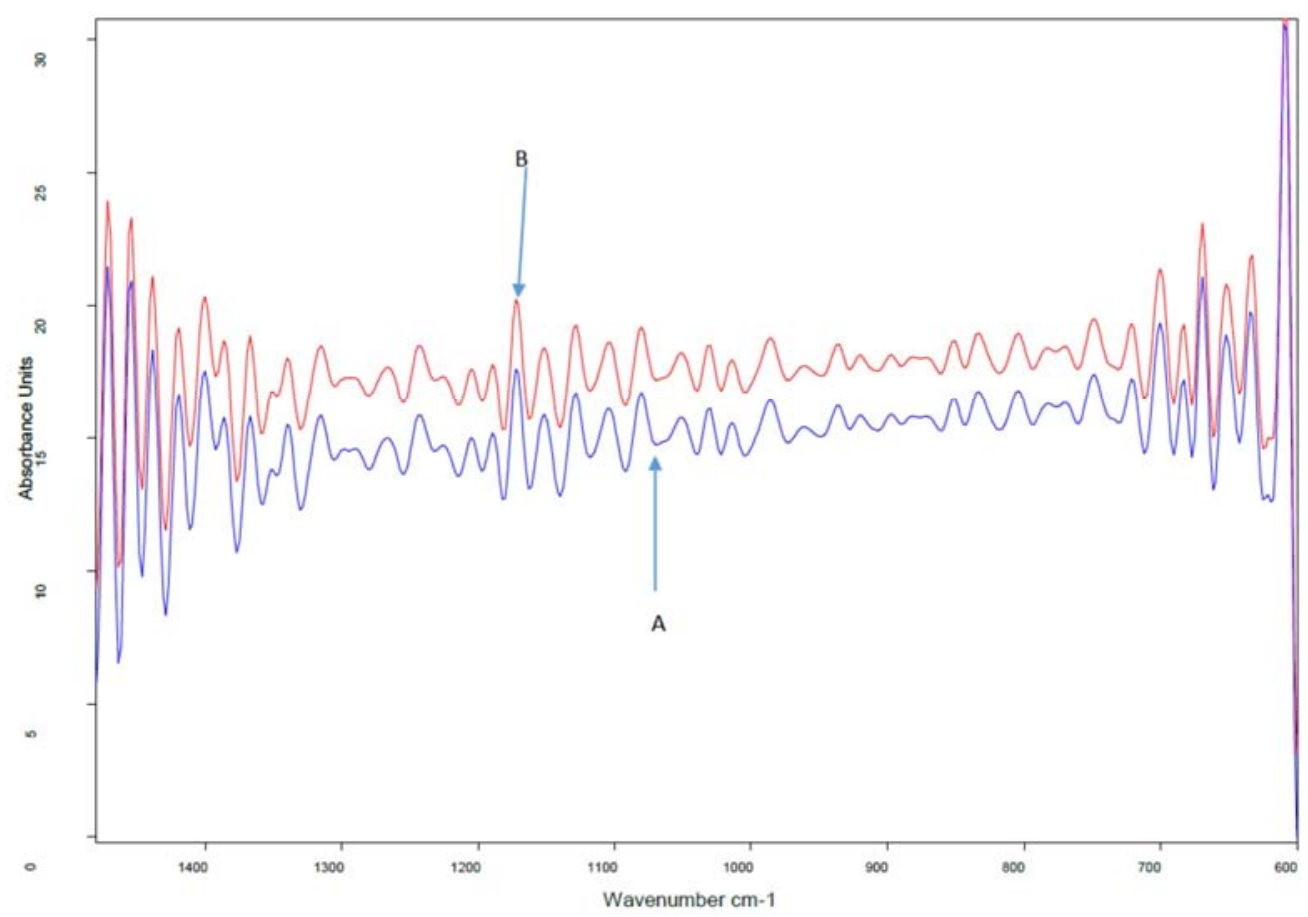

Figure 9. A- FSD spectra of HSA not exposed to magnetic field B- FSD spectra of HSA exposed to magnetic field. 


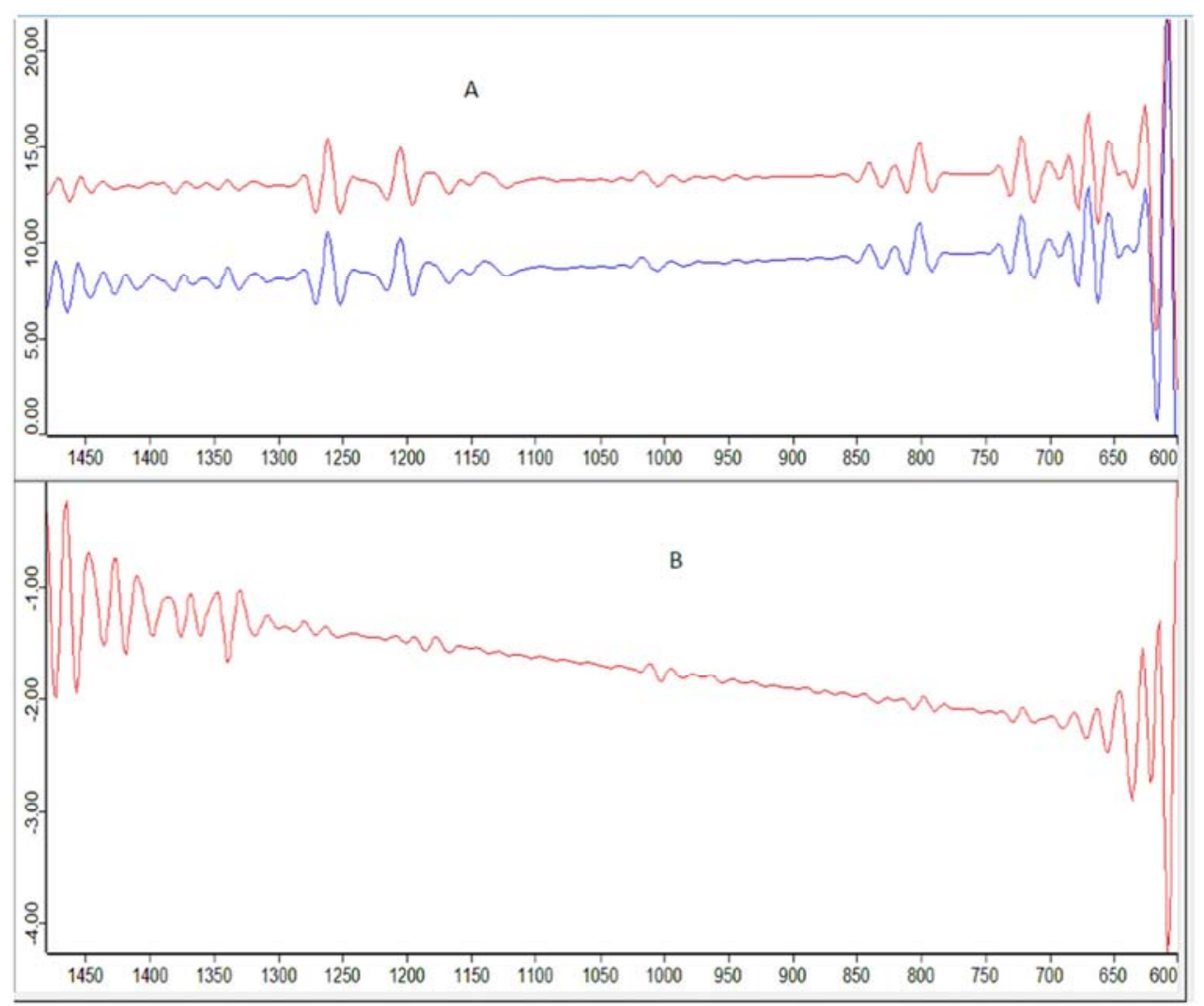

Wavenumbers $\mathrm{cm}^{-1}$

Figure 10. A: The FSD spectra for $\beta$-amyloid exposed to ELF $1.5 \mathrm{mT}$ (above) and not exposed (below). B: The difference between A $\beta$ exposed and not exposed to magnetic field.

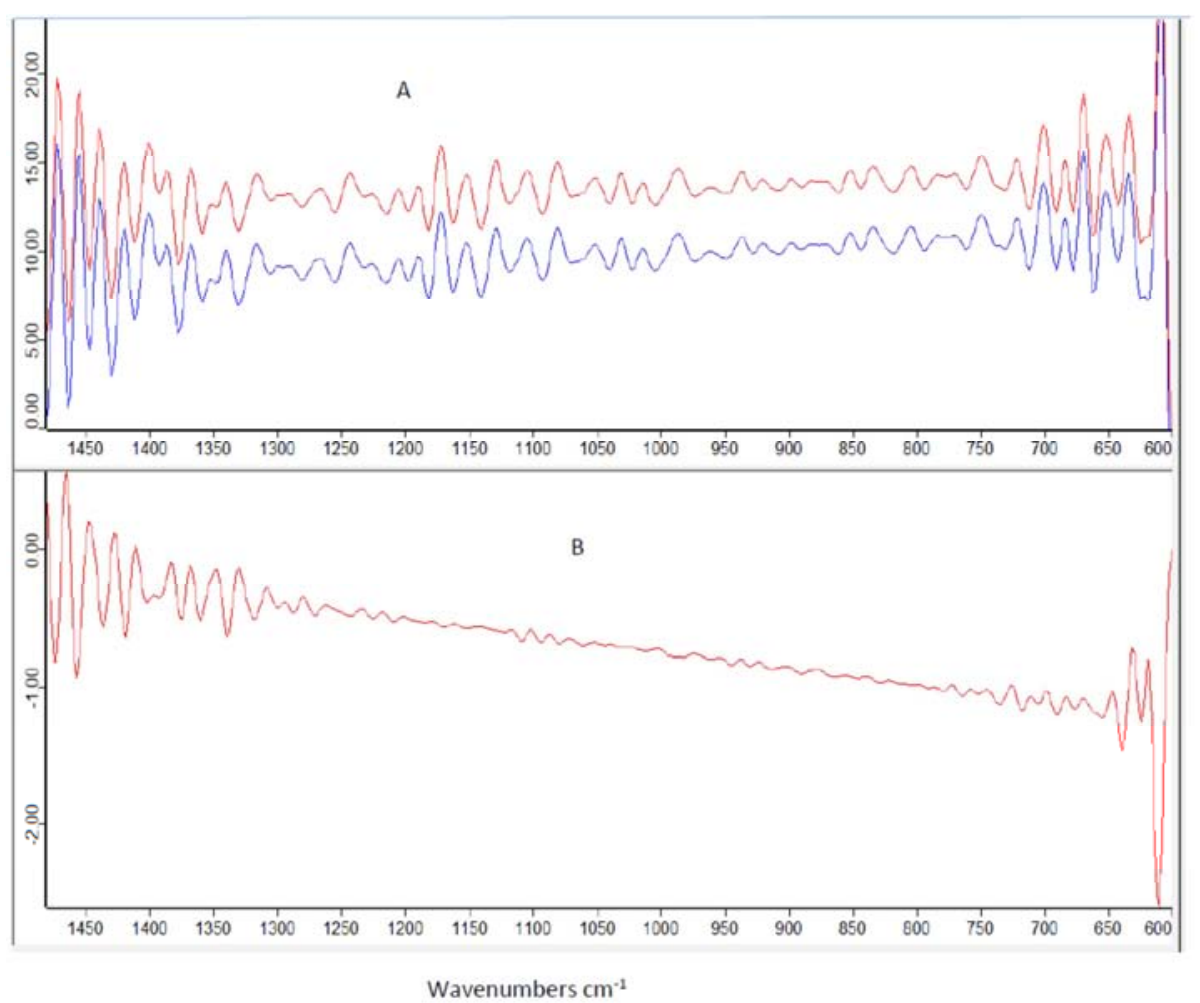

Figure 11. A-The FSD spectra of HSA exposed to ELF $1.5 \mathrm{mT}$ (above) and not exposed (below) B-The difference between HSA exposed and not exposed to magnetic field. 
Table 3. Peak positions of the absorbance spectra in the range (800-600) $\mathrm{cm}^{-1}$.

\begin{tabular}{|c|c|c|}
\hline \multicolumn{3}{|c|}{ A-Peak positions of the $A \beta$ absorption spectra } \\
\hline A $\beta$ (not exposed) & Exposed to magnetic field & Difference spectra \\
\hline 608 & 608 & --- \\
\hline --- & --- & 613 \\
\hline 625 & 625 & 626 \\
\hline 640 & 641 & 644 \\
\hline 653 & 653 & 661 \\
\hline 669 & 669 & 680 \\
\hline 685 & 685 & -- \\
\hline 700 & 700 & -- \\
\hline 721 & 721 & -- \\
\hline 739 & 739 & -- \\
\hline 782 & 782 & -- \\
\hline 800 & 800 & -- \\
\hline \multicolumn{3}{|c|}{ B-Peak positions of the HSA absorption spectra in the range } \\
\hline HSA (not exposed) & Exposed to magnetic field & Difference spectra \\
\hline 608 & 608 & -- \\
\hline 620 & 620 & 618 \\
\hline 633 & 633 & 629 \\
\hline 651 & 651 & 646 \\
\hline 670 & 670 & 669 \\
\hline 682 & 682 & 681 \\
\hline 700 & 700 & 697 \\
\hline 720 & 720 & 710 \\
\hline 749 & 749 & -- \\
\hline 784 & 784 & -- \\
\hline 804 & 804 & -- \\
\hline
\end{tabular}

The amide III and the fingerprint region are included in the range (1480-600) $\mathrm{cm}^{-1}$ have been treated in a similar way and the results are shown in figures: $8,9,10$ and 11 , while the observed changes are listed in tables 1 and 2 for the amide III bands, the observed changes in the fingerprint region are listed in table 3 .

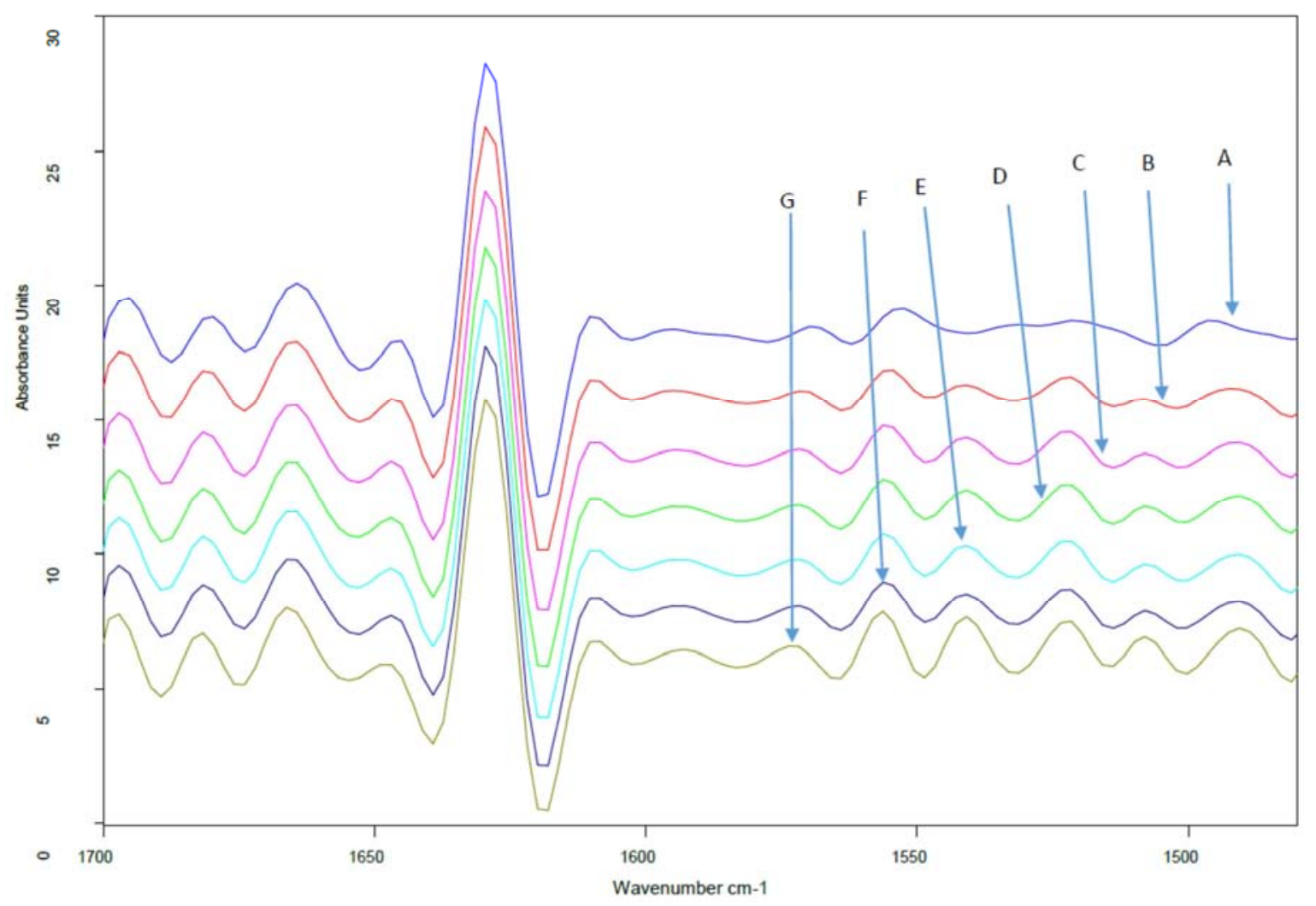

Figure 12. FSD spectra of $A \beta$ when (A- exposed to magnetic field of $1.5 \mathrm{mT})$, (B- 2 minutes after removal of field), (C- 4 minutes after removal), (D-6 minutes after removal), (E- 8 minutes after removal), ( $F$ - 10 minutes after removal), ( $G$ - No field). 


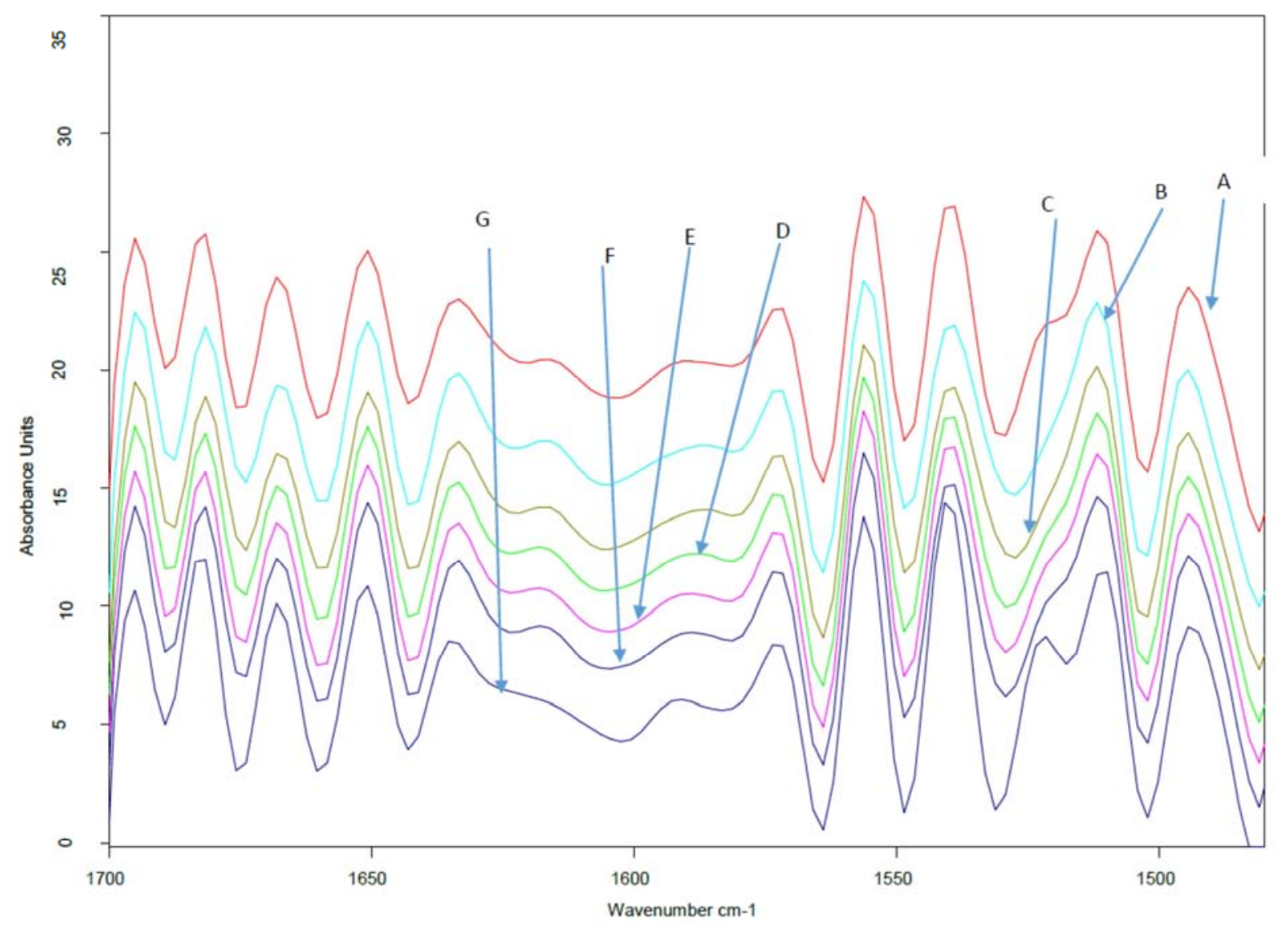

Figure 13. FSD spectra of HSA when (A-exposed to magnetic field), (B-2 minutes after removal of field), (C-4 minutes after removal), (D- 6 minutes after removal), (E- 8 minutes after removal), (F-10 minutes after removal), ( $G$ - before exposure to field).

The last part of the data is taken to investigate any possible remnant effect after removing the magnetic field on the samples. Each sample has been exposed to $1.5 \mathrm{mT}$ for 10 minutes, then after turning off the magnetic field the spectra is taken every 2 minutes for five consecutive readings. The FSD spectra is shown in figures 12 and 13 for the $A \beta$ and for the HSA samples respectively.

\section{Discussions}

In this work each of A $\beta$ and HSA samples' FTIR spectra is obtained before and after exposure to the ELF magnetic field without moving or changing the sample position. Sample stationary position makes comparison of intensities and peak positions more accurate and minimizes any errors due to sample motion. Changes in the FSD convoluted graphs in figure 4 indicate secondary structure changes as a result of $\mathrm{A} \beta$ exposure to magnetic field. These changes can be summarized as follows: the bands at $(1666,1648,1629$, and 1609) $\mathrm{cm}^{-1}$ have shown little or no increase of intensity, while the rest of the bands in the figure have shown a sharp decrease of intensity. The following bands (1508, 1522, 1556 , and 1573$) \mathrm{cm}^{-1}$ have shown large loss of intensity due to the effect of the magnetic field exposure. These remarks are also supported by figure 6-B, where the net effect of the magnetic field is shown to be strong near the above positions as listed in table 1 .
The FSD convoluted spectra in figure 5 indicate secondary structure changes upon HSA exposure to magnetic field. The majority of the bands in amide I and amide II lost intensity due to the magnetic effect at different proportionality with the exception of two bands at (1633, and 1616) $\mathrm{cm}^{-1}$. These two bands showed a little increase of their intensities. The shoulder at $1616 \mathrm{~cm}^{-1}$ has been enhanced strongly in intensity to become observable as peak by the effect of the magnetic field.

The peaks positions' changes for A $\beta$ and HSA are listed in tables 1 and 2 respectively. The positions' changes include various range of changes and while some peaks have shifted positions by larger amounts, others retained their energy positions. In general, the peak position can be correlated to the exact frequency of the bond vibrations of the particular amide region, which also relies on the nature of the hydrogen bonding with the amide group, and by the particular secondary structure adopted by the protein. The difference of the FSD spectra, plotted in figures 6-B and 7-B for $\mathrm{A} \beta$ and HSA respectively, show that all the peaks have been affected by the magnetic field at different levels. The peaks of the difference spectra show the frequencies at which maximum effect by the magnetic field occurred.

In general a peak in the difference spectra represents a gain of intensity due to magnetic field effect, which is caused by a constructive interference at that frequency of the peak position. While an inverted peak position represents a loss of intensity due to the magnetic field caused by a destructive 
interference at that frequency. If the difference spectra is a straight line, it indicates no contribution from the magnetic field implying no interference or energy exchange took place. A strong variations of the difference spectra in a particular region indicates a high degree of sensitivity to magnet effect in that region.

The quenching effect on the spectra of amide II region was stronger than the quenching effect on the spectra in the amide I region. [47] This difference indicates that the magnetic field has more damping influence on the out of phase combination of $\mathrm{N}-\mathrm{H}$, in-plane bending, and $\mathrm{C}-\mathrm{N}$ stretching vibrations modes than on the in-phase combination of $\mathrm{C}=\mathrm{O}$ stretching vibrations coupled to the $\mathrm{C}-\mathrm{N}$ stretching.

The changes in the FSD spectra in the region (1480 1220) $\mathrm{cm}^{-1}$ which includes the amide III band are shown in figures 8 and 9 for $A \beta$ and HSA respectively. The peak positions for this part of the spectra are also summarized in table 1 for A $\beta$ bands and in table 2 for the HSA bands.

The changes in the FSD spectra of $A \beta$ for the range of (1220 to 600) $\mathrm{cm}^{-1}$ are shown also in figures 8 and 10 however, the peak positions are summarized in table 3 -A. It seems that most of the peaks show very little or no change in terms of their peak positions and in their intensities.

The difference spectra in figure 10-B, yields very little variations indicating little or no effect being imposed on most of the peak positions and their intensities by the magnetic field. The main changes are limited to the appearance of a new peak at $613 \mathrm{~cm}^{-1}$, and few peaks changed positions as follows: $(625 \rightarrow 626,640 \rightarrow 644$, and $653 \rightarrow 661) \mathrm{cm}^{-1}$.

The changes in the FSD spectra for the HSA in the range $(1220-600) \mathrm{cm}^{-1}$ are shown in figures 9 and 11 and the peak positions are listed in table 3-B. Most bands show little or no change in their peak positions and in their relative intensities due to the exposure to the magnetic field.

The difference spectra in figure 11-B shows peak positions shift as follows: $(633 \rightarrow 629,651 \rightarrow 646,670 \rightarrow 669,682 \rightarrow 681$, $700 \rightarrow 697$, and $720 \rightarrow 710) \mathrm{cm}^{-1}$. In addition to a strong magnetic field effect on band intensity occurring at $(618,629$ and 646) $\mathrm{cm}^{-1}$ and very little effect on all other bands.

This implies that for both proteins the absorption bands in amide IV region $(625-765) \mathrm{cm}^{-1}$, which are mainly caused by combinations of OCN bending vibrations mixed with out of phase N-H bending $[48,49]$ are highly sensitive to magnetic field effect. It is interesting to note that both regions amide II and amide IV show sensitivity to magnetic field exposure, and both bands caused by out of phase combinations of N-H bending mixed with OCN bending or stretching. One may speculate that out of phase combination of molecular vibrations leave molecules more susceptible to be twisted by the magnetic field.

The qualitative analysis of the secondary structure for $A \beta$ and HSA proteins is based on the observed changes in peak position, band intensity and band width for the individual bands. It is factually accepted that general vibrational spectroscopy is highly sensitive and can detect any small change $(0.02 \%)$ in bond strength [50]. Any changes in bond strength or the number of bonds is reflected in a change of the band relative intensity.

Our experimental results show a large loss of intensity to most of the bands in the amide II region for both $A \beta$ and HSA and a small decrease of intensity to some of the bands in amide I. The loss of intensity implies that exposure to magnetic field introduces a damping factor which alters molecular vibrations at the bonding sites. This may be explained by a destructive interference imposed by the magnetic field on the out of phase combinations of $\mathrm{C}-\mathrm{N}$ stretching and $\mathrm{N}-\mathrm{H}$ bending. On the other hand, the magnetic field did not interfere with the in- phase combinations of N-H bending and $\mathrm{C}-\mathrm{N}$ stretching in the amide III region and minor changes were observed on its bands' intensities.

The interference of the magnetic field with molecular vibrations can be caused by the Hall Effect. Free charges tend to move by the magnetic force and the positive charges accumulate on one side while the negative charges accumulate at the opposite side creating region with potential difference. The accumulated charges on the opposing sides exert opposing forces on the ends of the vibrating molecule and produce a twisting effect on its vibration.

In the presence of a magnetic field in a medium several forces can influence the motion of charged particle. For example Lorentz force $(\mathrm{F}=\mathrm{qv} \mathrm{X} \mathrm{B})$, and the magnetic gradient force $\left(F=\left(p_{m} \nabla\right) B\right)$, where $q$ and $v$ are the charge and the velocity of the particle, $\mathrm{B}$ is the magnetic induction, and $\mathrm{p}_{\mathrm{m}}$ is the dipole magnetic moment. These forces can have an impact but to a lesser degree than the impact of electric forces [51]. When the vibrations of the atoms are out of phase, these forces may produce a stronger torque on the molecules than the vibrations of the in-phase combinations case. This may explain why the peak positions of amide II bands are shifted to higher energies while at the same time lost some of their intensities. Physically these bonds are becoming stiffer with a larger spring force constant due to the twisting effect and losing intensity due to the damping effect by the Hall Effect. It is suggested that twisting of antiparallel beta sheets can also leads to a shift to higher energy by previous studies [52, 53].

These bands whose peaks shifted to a higher energy indicate a change in the energy of molecular vibration due to the increase in the spring restoring force, which is caused by additional hydrogen bonding. It is well known that hydrogen bonds help in stabilizing proteins which is needed for catalysis. Therefore, additional hydrogen bonds can lower the frequency of a stretching vibration. On the other hand, less hydrogen bonding decreases the strain on the molecules can shift the peak position to lower energy [54]. It is suggested that each of the two possible hydrogen bonds to the C-O group lowers the amide I frequency by 20 to $30 \mathrm{~cm}^{-1}$ and a hydrogen bond to the NH group by 10 to $20 \mathrm{~cm}^{-1}[55,56]$.

The small increase in the intensity of $\beta$-sheets in amide I for $A \beta$ spectra at $1629 \mathrm{~cm}^{-1}$ and for HSA spectra (1635 and 1616) $\mathrm{cm}^{-1}$ occurred after ELF magnetic field exposure can be attributed to disorder and unfolding processes in the structure of the protein [57-59].

The peak positions of the bands in amide I and amide III 
are hardly affected by the number of amide groups in the strand of the sheet, but depends on the number of strands. Sheets with a larger number of strands have a lower spectral position of the main band $[52,60]$.

The effect of exposing A $\beta$ and HSA to ELF magnetic field is examined further by monitoring spectra changes after removing the magnetic fields. Figures 12 and 13 show the spectra changes as a function of time. It seems that after removing the field, the affected absorbance intensities and the shift of the band positions takes on a gradual change slowly returning to their control levels, obtained before the exposure. The changes in the spectra are monitored every two minutes, but it is clearly evident that these two proteins need more than 10 minutes to revert to their initial control states.

This behaviour implies that the conformational changes on the secondary structure of the proteins are not momentarily, and the proteins' exposure to the magnetic field leads to internal energy changes in the proteins. Exposure to magnetic field can cause charge motion and induce Eddy currents within the protein. But these new currents induce a new magnetic field to counter the effect of the original magnetic field according to Lenz's law. Therefore, the protein may act as an inductor-capacitor circuit which causes energy oscillation and that leads to a time delay for a full recovery and the return to the original state.

\section{Conclusion}

The experimental results showed that $\mathrm{A} \beta$ and HSA are sensitive to ELF magnetic field exposure and the following changes have been observed: (1) Peak positions' shifts and intensity changes for amide II bands were highly noticeable. (2) A small decrease in band intensities of antiparallel $\beta$ sheets and $\beta$-turns in the amide I has been detected versus a small increase of intensities of $\alpha$-helix and parallel $\beta$-sheets in that region. (3) No major changes in the amide III region have been observed (4) the region between (720-600) $\mathrm{cm}^{-1}$ has shown high sensitivity to ELF magnetic fields, and (5) Recovery of the magnetic effects are not instantaneous.

This study suggests that ELF magnetic fields can interact with proteins and may promote biological tasks or cause a dysfunction for the protein depending on whether the exchanged energy is appropriate for a particular task or not.

\section{Acknowledgements}

This work is supported by the German Research Foundation DFG Grant No. DR228/24-2.

\section{References}

[1] A. Elliot and E. J. Ambrose, "Structure of Synthetic Polypeptides," Nature, 165, (1950) 921-922.

[2] T. Miyazawa, 'Infrared Spectra and Helical Conformations', in "Poly-a- amino Acids," ed. G. D. Fasman, Marcel Dekker, New York, (1967) 69-103.
[3] S. Krimm and J. Bandekar, "Vibrational spectroscopy and conformation of peptides, polypeptides, and proteins," Adv. Protein Chem., 38, (1986) 181-364.

[4] Byler DM, Susi H., "Examination of the secondary structure of proteins by deconvolved FTIR spectra," Biopolymer, 25 (1986) 469-487.

[5] Surewicz, W. K., Mantsch, H. H. and Chapman, D., "Determination of Protein Secondary Structure by Fourier Transform Infrared Spectroscopy: A Critical Assessment," Biochemistry, 32 (1993) 389-394.

[6] M. X. Xie, Y. Liu, "Studies on amide III infrared bands for the secondary structure determination of proteins," Chem. J. Chin. Univ.-Chinese 24 (2003) 226-231.

[7] S. Cai, B. R. Singh, "Identification of ß-turn and random coil amide III infrared bands for secondary structure estimation of proteins," Biophys. Chem. 80 (1999) 7-20.

[8] S. Cai, B. R. Singh, "A distinct utility of the amide III infrared band for secondary structure estimation of aqueous protein solutions using partial least squares methods," Biochemistry 43 (2004) 2541-2549.

[9] R. J. Ellis and F. U. Hartl, "Principles of protein folding in the cellular environment," Current Opinion in Structural Biology, vol. 9, no. 1 (1999) 102-110.

[10] M. J. Gething and J. Sambrook, "Protein folding in the cell," Nature, vol. 355, no. 6355 (1992) 33-45.

[11] Stroud, J. C., Liu, C., Teng, P. K. and Eisenberg, D. (2012) Toxic Fibrillar Oligomers of Amyloid- $\beta$ Have Cross$\beta$ Structure. Proceedings of the National Academy of Sciences of the United States of America, 109, 7717-7722.

[12] Lu, M., Hiramatsu, H., Goto, Y. and Kitagawa, T., "Structure of Interacting Segments in the Growing Amyloid Fibril of $\beta 2$ Microglobulin Probed with IR Spectroscopy," Journal of Molecular Biology, 362 (2006) 355-364.

[13] Saqer M. Darwish, Shurook Y. Aiaidah, Imtiaz M. Khalid, Musa M. Abuteir, Lena Qawasmi, "Spectroscopic Investigations of $\beta$-Amyloid Interactions with Propofol and LArginine," Open Journal of Biophysics, 2015, 5,(2015) 50-67.

[14] T. Peters, "Structure of serum albumin,"Adv. Protein Chem. 37 (1985) 161-245.

[15] Santoro N, Lisi A, Pozzi D, Pasquali E, Serafino A, GrimaldiS.,"Effect of extremely low frequency (ELF) magnetic field exposure on morphological and biophysical properties of human lymphoid cell line (Raji)," Biochim. Biophys. Acta, 1357 (1997) 281-290.

[16] Toshitaka Ikehara, Hisao Yamaguchi, Keiko Hosokawa, Hiroshi Miyamoto, Katsuo Aizawa, "Effects of ELF magnetic field on membrane protein structure of living Hela cells studied by Fourier transform infrared spectroscopy," Bioelectromagnetics 24 (2003) 457-464.

[17] WHO, "Extremely low frequency (ELF) fields," Environmental Health Criteria 35, World Health Organization, Geneva, Switzerland, 1984.

[18] Emanuele Calabro and Salvatore Magazu, "Electromagnetic Fields Effects on the Secondary Structure of Lysozyme and Bioprotective Effectiveness of Trehalose," Advances in Physical Chemistry, 2012 (2012) 1-6. 
[19] S. Magazu, E. Calabro, and S. Campo, "FTIR spectroscopy studies on the bioprotective effectiveness of trehalose on human hemoglobin aqueous solutions under $50 \mathrm{~Hz}$ electromagnetic field exposure," J. Physical Chemistry B, 114, no. 37 (2010) 12144-12149.

[20] Mehmet Zulkuf Akdag, Suleyman Dasdag, Dilek Ulker Cakir, Beran Yokus, Goksel Kizil \& Murat Kizil, Do 100- and 500mT ELF magnetic fields alter beta-amyloid protein, protein carbonyl and malondialdehyde in rat brains?, Electromagnetic Biology and Medicine, 32, 3 (2013) 363-372.

[21] Marcella Reale, Mohammad A. Kamal, Antonia Patruno, Erica Costantini, Chiara D' Angelo, Miko Pesce, Nigel H. Greig, Neuronal Cellular Responses to Extremely Low Frequency Electromagnetic Field Exposure: Implications Regarding Oxidative Stress and Neurodegeneration, PLOS One, 9, 8 (2014) doi: 10.1371.

[22] Greenland S, Sheppard AR, Kaune WT, Poole C, Kelsh MA. 2000. A pooled analysis of magnetic fields, wire codes, and childhood leukemia. Epidemiology 11, 624-634.

[23] Ahlbom A, et al. 2000. A pooled analysis of magnetic fields and childhood leukaemia. Br. J. Cancer 83, 692-698.

[24] International Agency for Research on Cancer 2002. Static and extremely low-frequency (ELF) electric and magnetic fields. IARC monographs on the evaluation of carcinogenic risks to humans, vol. 80 Lyon, France: IARC.

[25] National Radiological Protection Board 2001. ELF electromagnetic fields and the risk of cancer. Report of an Advisory Group on Non-ionising Radiation. Documents of the NRPB, vol 12 Chilton, Oxon, UK: NRPB.

[26] Liedvogel M, Mouritsen H. 2010. Cryptochromes: a potential magnetoreceptor: what do we know and what do we want to know? J. R. Soc. Interface 7, S147-S162.

[27] Ritz T, Adem S, Schulten K., "A model for photoreceptorbased magnetoreception in birds," Biophys. J. 78 (2000) 707 718 .

[28] Shubhajit Paul, Alexey S. Kiryutin, Jinping Guo, Konstantin L. Ivanov, Jörg Matysik, Alexandra V. Yurkovskaya \& Xiaojie Wang, Magnetic field effect in natural cryptochrome explored with model compound, Scientific Reports 7: 11892 |DOI: 10.1038/s41598-017-10356-4 (2017).

[29] Yong E., "Flying sly: supersensory perception," New Sci. 2788 (2010) 42- 45.

[30] Ball P.," Physics of life: the dawn of quantum biology," Nature 474 (2011) 272-274.

[31] Hoff AJ.," Magnetic field effects on photosynthetic reactions," Q. Rev. Biophys. 14 (1981) 599-665.

[32] Van Dijk B, Carpenter JKH, Hoff AJ, Hore PJ.," Magnetic field effects on the recombination kinetics of radical pairs," J. Phys. Chem. B 102 (1988) 464-472.

[33] Liu Y, Edge R, Henbest K, Timmel CR, Hore PJ, GastP.,"Magnetic field effect on singlet oxygen production in a biochemical system," Chem. Commun. 2 (2005) 174-176.

[34] Alex R. Jones, magnetic field effects in proteins, Molucular Physics. 14, 11 (2016) 1691-1702.

[35] Emrys W. Evans, Charlotte A. Dodson, Kiminori Maeda, Till Biskup, C. J. Wedge, Christiane R. Timmel, Magnetic field effect on flavoproteins and related systems, Interface Focus, 3 (5) 2013: 20130037. doi: 10.1098/rsfs.2013.0037.

[36] Saqer M. Darwish, Sawsan E. Abu sharkh, Musa M. Abu Teir, Sami A. Makharza, Mahmoud M. Abu-hadid," Spectroscopic investigations of pentobarbital interaction with human serum albumin," Journal of Molecular Structure 963 (2010) 122-129.

[37] R. K. Dukor, J. M. Chalmers, P. R. Griffiths (Eds.), Vibrational Spectroscopy in the Detection of Cancer, Handbook of Vibrational Spectroscopy, vol. 5, Wiley, Chichester, 2001 (Chapter 3).

[38] Sirotkin, V. A., Zinatullin, A. N., Solomonov, B. N., Faizullin, D. A. and Fedotov, V. D.," Calorimetric and Fourier transform Infrared Spectroscopic Study of Solid Proteins Immersed in Low Water Organic Solvents," Biochimicaet Biophysica Acta, 1547 (2001) 359-369.

[39] Y. N. Chirgadze, O. V. Fedorov, N. P. Trushina," Secondary structure of $\mathrm{Na}^{+}, \mathrm{K}^{+}$-dependent adenosine triphosphatase," Biopolymers 14 (1975) 679-694.

[40] Cerf, E., et al., "Antiparallel $\beta$-Sheet: A Signature Structure of the Oligomeric Amyloid $\beta$-Peptide," Biochemical Journal, 421 (2007) 415-423.

[41] Sarroukh, R., Cerf, E., Derclaye, S., Dufrêne, Y. F., Goormaghtigh, E., Ruysschaert, J. M., Raussens, V., "Transformation of Amyloid $\beta(1-40)$ Oligomers into Fibrils Is Characterized by a Major Change in Secondary Structure," Cell. Cellular and Molecular Life Sciences, 68 (2011) 1429-1438.

[42] Dong A, Huang P, Caughey WS., "Redox-dependent changes in $\beta$-extended chain and turn structures of cytochrome $\mathrm{c}$ in water solution determined by second derivative amide I infrared spectra. Biochemistry," 31 (1992) 182-189.

[43] Susi H, Byler DM.," Resolution-enhanced fourier transform infrared spectroscopy of enzymes," Methods Enzymol, 130 (1986) 290-311.

[44] Kong, J. and $\mathrm{Yu}, \mathrm{S} .$, "Fourier Transform Infrared Spectroscopic Analysis of Protein Secondary Structures,"Acta Biochimicaet Biophysica Sinica, 39 (2007) 549-559.

[45] G. Deleris, C. Petibios, Vib. Spectrosc. 32 (2003) 129-136.

[46] E. Bramanti, E. Benedetti, "Determination of the secondary structure of isomeric forms of human serum albumin by a particular frequency deconvolution procedure applied to Fourier transform IR analysis," Biopolymers 38 (1996) 639653.

[47] Emanuele Calabro and Salvatore Magazu, Unfolding-induced in Haemoglobin by Exposure to Electromagnetic Fields: A FTIR Spectroscopy Study, Orient. J. Chem., Vol. 30 (1), (2014) 31-35.

[48] Heinz Fabian and Werner Mantele, "inferred spectroscopy of proteins, Biochemical applications", John Wiley \&son's ltd. 2002.

[49] Patrick Garidel and Heidrun Schott, Fourier-Transform Midinfrared spectroscopy for analysis and screening of liquid protein formulations, Bioprocess international 40 (2006) 4855 .

[50] H. Deng, R. Callender, "Raman spectroscopic studies of the structures, energetics, and bond distortions of substrates bound to enzymes," Methods Enzymol. 308 (1999) 176-201. 
[51] V Zablotskii, O Lunov, S Kubinova, T Polyakova, E Sykova and A Dejneka, Effects of high-gradient magnetic fields on living cell machinery, J. Phys. D: Appl. Phys. 49 (2016) 1-23.

[52] Kubelka, T. A. Keiderling," The anomolous infrared amidei intensity distribution in $13 \mathrm{C}$ isotopically labelled peptide," J. Am. chem. soc. 123 (2001) 6142-6150.

[53] Andreas Barth, "IR spectroscopy of proteins," Biochimicaet Biophysica Acta, 1767 (2007) 1073-1101.

[54] N. P. Colthump, L. H, Daly, S. B. Wiberley, Introduction to Inferred and Raman Spectroscopy 2nd ed. Academic press. New York 1975.

[55] H. Torri, T. Tatsumi," Effects of hydrogen on the structure vibrational wavenumbers, vibrational force field and resonance Raman intensities of N-methylacetamide," J. Raman spectroscopy, 29 (1998) 537-546.

[56] B. Mennucci, J. M. Martinez," How to model solavation of piptides? Insights from quantum Mechanical and molecular dynamics study of N-methylacetamide I," J. phys. chem. 109 (2005) 9818-9829.
[57] P. Huang, A. Dong, and W. S. Caughey, "Effects of dimethyl sulfoxide, glycerol, and ethylene glycol on secondary structures of cytochrome c and lysozyme as observed by infrared spectroscopy," Journal of Pharmaceutical Sciences, 84, no. 4 (1995) 387-392.

[58] R. Bauer, R. Carrotta, C. Rischel, and L. Øgendal, "Characterization and isolation of intermediates in $\beta$ lactoglobulin heat aggregation at high $\mathrm{pH}$," Biophysical Journal, vol. 79, no. 2 (2000) 1030-1038.

[59] S. Magazu, E. Calabro, and S. Campo, "Studying the electromagnetic-induced changes of the secondary structure of bovine serum albumin and the bioprotective effectiveness of trehalose by FTIR spectroscopy," Journal of Physical Chemistry B, vol. 115, no. 21 (2011) 6818-6826.

[60] Yu. N. Chirgadze, N. A. Nevskaya Infrared spectra and resonance interaction of amide-I vibration of the antiparallelchain pleated sheet, biopolymer, Volume 15, Issue 4 (1976) 607-625. 